\title{
The ubiquitin-specific protease 17 is involved in virus-triggered type I IFN signaling
}

\author{
Rui Chen ${ }^{1}$, Lu Zhang ${ }^{1}$, Bo Zhong ${ }^{1}$, Bo Tan ${ }^{1}$, Yu Liu ${ }^{1}$, Hong-Bing Shu ${ }^{1}$ \\ ${ }^{1}$ College of Life Sciences, Wuhan University, Wuhan 430072, China
}

Viral infection initiates a series of signaling cascades that activate the transcription factors nuclear factor kappa $B$ and interferon regulatory factor 3 , which collaborate to induce transcription of genes for type I interferons (IFNs) and other cytokines. Here we report that the deubiquitinating enzyme ubiquitin-specific protease 17 (USP17) is required for virus-induced RIG-I- and melanoma differentiation-associated protein-5 (MDA5)-mediated type I IFN signaling. Knockdown of endogenous USP17 inhibited virus-, cytoplasmic poly(I:C)- and poly(dA:dT)-induced activation of the IFN- $\beta$ promoter and cellular antiviral responses. We further found that knockdown of USP17 inhibited RIG-I- and MDA5-induced but not downstream activator-induced activation of the IFN- $\beta$ promoter, which was correlated with an increase in ubiquitination levels of RIG-I and MDA5. Taken together, our findings suggest that USP17 functions through deubiquitination of RIG-I and MDA5 to regulate virus-induced type I IFN signaling.

Keywords: USP17; RLR; deubiquitination; type I IFNs

Cell Research (2010) 20:802-811. doi:10.1038/cr.2010.41; published online 6 April 2010

\section{Introduction}

Viral infection triggers a series of signaling events that lead to induction of type I interferons (IFNs), including IFN- $\beta$ and IFN- $\alpha$ family cytokines [1-3]. Type I IFNs initiate a series of signaling cascades, leading to transcriptional activation of a wide range of genes. The products of these genes collaborate to orchestrate inhibition of viral replication or clearance of virus-infected cells, leading to innate antiviral responses [1-3]. Therefore, type I IFNs play central roles in host defense against invading viruses.

Studies during the past decade have revealed a working model on virus-triggered type I IFN signaling. Viral infection generates double-stranded RNA (dsRNA) and

\footnotetext{
Correspondence: Hong-Bing Shu

Tel: +86-27-68753795; Fax: +86-27-68753780

E-mail: shuh@whu.edu.cn

Abbreviations: CARD (caspase recruitment domain); DCs (dendritic cells); IKK $\varepsilon$ (inhibitor of nuclear factor kappa-B kinase subunit epsilon); IRF-3 (interferon regulatory factor 3); ISRE (IFN-stimulated response element); MDA5 (melanoma differentiation-associated protein-5); NF-кB (nuclear factor kappa B); RIG-I (retinoic acid-inducible gene I); TBK1 (TANKbinding kinase 1); VISA (virus-induced signaling adaptor)

Received 19 November 2009; revised 1 February 2010; accepted 22 February 2010; published online 6 April 2010
}

5' triphosphorylated single-stranded RNA (5'pppssRNA), which are recognized by retinoic acid-inducible gene I (RIG-I)-like receptors (RLRs) and Toll-like receptors (TLRs) [4-6]. The receptors then undergo conformational changes and recruit downstream adaptor proteins and kinases, leading to activation of transcription factors such as nuclear factor kappa $\mathrm{B}(\mathrm{NF}-\kappa \mathrm{B})$ and interferon regulatory factor 3 (IRF3) that collaborate to induce production of type I IFNs. For example, engagement of TLR3 by dsRNA triggers TRIF-mediated signaling. TRIF on the one hand recruits TRAF6/RIP and inhibitor of nuclear factor kappa-B kinase subunit alpha (IKK $\alpha) / \beta / \gamma$ complex for activation of $N F-\kappa B$, and on the other hand, recruits TRAF3 and TANK-binding kinase 1 (TBK1)/IKK $\varepsilon$ for activation of IRF3 [7-10]. The RLR family cytoplasmic receptors RIG-I and melanoma differentiation-associated protein-5 (MDA5) bind to viral RNA and are recruited to the mitochondrial adaptor protein virus-induced signaling adaptor (VISA, also known as MAVS, IPS-1 and Cardif) [11-14]. VISA interacts with MITA (also called STING) and TRAF3, and the VISA-MITA-TRAF3 complex provides a platform for TBK1-mediated phosphorylation of IRF3 $[15,16]$. VISA is also associated with TRAF6, which activates NF- $\kappa \mathrm{B}$ through the IKK complex [11]. Recently, it has been demonstrated that RNA polymerase III recognizes invading viral DNA and AT-rich dsDNA 
(poly(dA:dT)) and transcribes them into RNAs with the 5'ppp structure, which initiates RIG-I-mediated signaling $[17,18]$.

Deubiquitinating enzymes (DUBs) are proteases that cleave ubiquitin or ubiquitin-like proteins from proproteins or conjugates with target proteins. About 100 putative DUBs are encoded by the human genome, and they belong to five different families [19], including ubiquitinspecific proteases, carboxy-terminal hydrolases, otubain proteases, the Machado-Joseph disease protease and the metallo-proteases referred to as JAMM. Like ubiquitination, deubiquitination is a highly regulated process that has been implicated in numerous cellular functions, including cell cycle regulation [20], proteaosome- and lysosome-dependent protein degradation [21-23], gene expression [24], signal transduction [25], kinase activation [26], and more [19, 27].

Virus-induced type I IFN signaling pathways are tightly controlled by ubiquitination and deubiquitination. Several ubiquitin ligase enzymes have been identified to regulate these processes [28-35]. However, only a few DUBs are known to regulate these pathways: A20 was shown to negatively regulate the RIG-I-induced antiviral state [36], DUBA was identified to be required for efficient deubiquitination of TRAF3 and to function as a negative regulator of innate immune responses [25], and CYLD, known as a tumor suppressor, was recently reported to negatively regulate the activation of TBK1/ IKK $\varepsilon$ [37].

In this study, we identified that the ubiquitin-specific protease 17 (USP17, also called DUB3) was required for virus-triggered expression of type I IFNs by screening a cDNA library encoding DUBs. Knockdown of USP17 by RNAi inhibited Sendai virus (SeV)-triggered activation of IRF3, production of IFN- $\beta$ and cellular antiviral response. In addition, knockdown of USP17 enhanced the ubiquitination levels of RIG-I and MDA5 and reduced SeV-induced expression of RIG-I and MDA5. Our data collectively provide evidence that the USP family member USP17 is required in virus-triggered type I IFN signaling.

\section{Results}

Identification of USP17 as a regulator of virus-induced type I IFN signaling

To identify potential DUBs that regulate virus-triggered signaling, we screened a pool of $40 \mathrm{cDNA}$ clones encoding DUBs obtained from Origene and constructed in our laboratory for their abilities to regulate $\mathrm{SeV}$-induced activation of the IFN- $\beta$ promoter in reporter assays in 293 cells. This effort identified USP17 when overex- pressed as a potent inhibitor of $\mathrm{SeV}$-induced activation of the IFN- $\beta$ promoter.

USP17 has been demonstrated to be expressed ubiquitously in various tissues and cells and to play important roles in cell proliferation and viability $[38,39]$. To confirm the effect of USP17 in virus-induced innate immune response, we made independent mammalian expression plasmids for USP17. In reporter assays, USP17 expression strongly inhibited Sev-induced activation of the IFN- $\beta$ promoter (Figure 1A and Supplementary information, Figure S1). In similar assays, we found that overexpression of USP17 inhibited SeV-induced activation of NF- $\kappa \mathrm{B}$ and IFN-stimulated response element (ISRE) (Figure $1 \mathrm{~B}$ and $1 \mathrm{C}$ ) and $\mathrm{TNF} \alpha$-induced activation of $\mathrm{NF}-\kappa \mathrm{B}$ (Figure 1C), suggesting that overexpression of USP17 regulates various signaling pathways.

Because USP17 is a deubiquitination enzyme, we determined whether USP17 mediates deubiquitination of molecules involved in virus-triggered signaling. Unexpectedly, we found that USP17 overexpression strongly abolished the ubiquitination levels of all examined proteins, such as MDA-5, RIG-I, VISA, MITA, IKK $\varepsilon$ and TRAF3 (Figure 1D). The abolishment of polyubiquitin chains conjugated to these proteins is caused by the nonspecific cleavage effect of overexpression of USP17, as evidenced by the fact that USP17 abolished the ubquitination of cellular proteins and/or cytosolic synthesized polyubiquitin chains, whereas USP5 or DUBA did not (Figure 1E). These results suggest that overexpression of USP17 exhibits global and most probably nonspecific deubiquitination enzymatic activities towards various cellular proteins and/or polyubiquitin chains.

\section{USP17 is required for virus-induced type I IFN signaling}

Because overexpression of USP17 exhibited nonspecific enzymatic activity, we examine the physiological roles of USP17 in virus-triggered signaling. We made three RNAi expression vectors containing different sequences targeting the human USP17 mRNA. Transient transfection and immunoblot analysis indicated that these vectors could inhibit expression of both overexpressed and endogenous USP17 (Figure 2A).

Interestingly, these three USP17 RNAi vectors markedly inhibited SeV-induced activation of the IFN- $\beta$ promoter in reporter assays in 293 cells, and the degrees of inhibition were correlated to the knockdown efficiencies of USP17 expression by each RNAi vector (Figure 2B). (The \#3 USP17-RNAi plasmid was used for all the experiments described below if not specified otherwise. Similar results were obtained with the other USP17RNAi plasmids.) Consistent with these observations, knockdown of USP17 inhibited SeV-induced transcrip- 
A

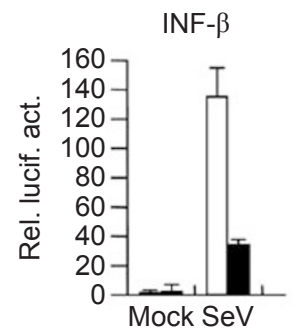

D

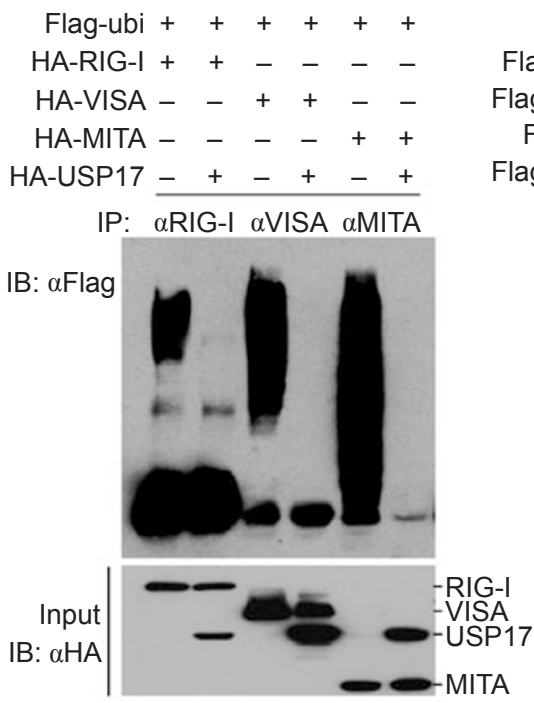

B

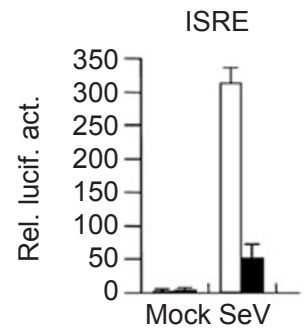

HA-ubi ++++++

Flag-IKK $\varepsilon----++$

lag-USP17 - + +-+

IP: $\alpha$ MDA5 $\alpha$ TRAF3 $\alpha \mathrm{IKK} \varepsilon$

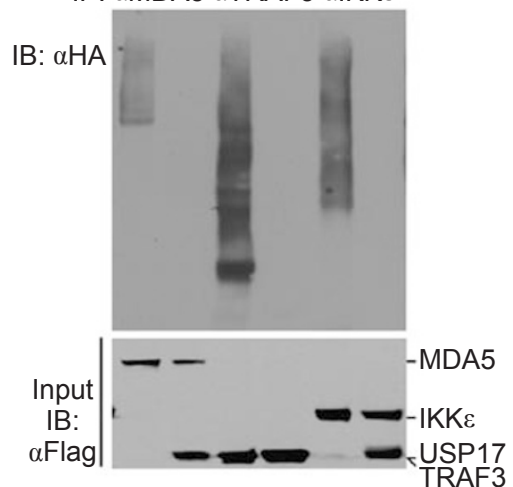

C

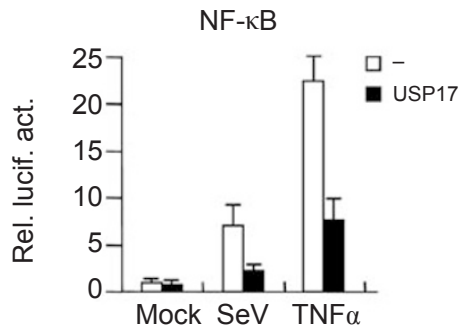

E
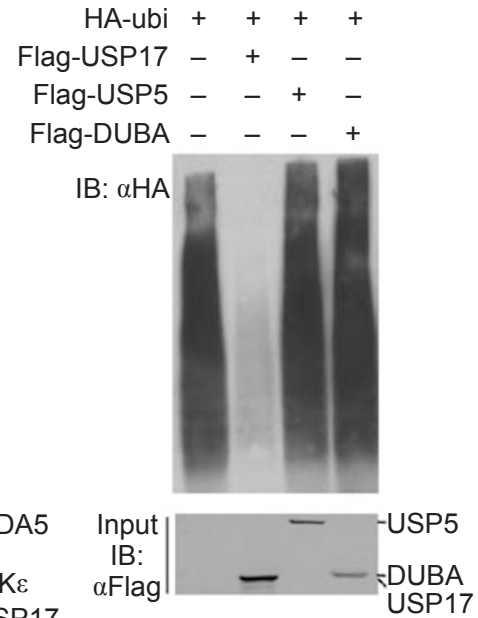

Figure 1 USP17 inhibits virus-induced type I IFN signaling. (A) USP17 inhibits SeV-induced activation of the IFN- $\beta$ promoter. 293 cells $\left(1 \times 10^{5}\right)$ were transfected with an IFN- $\beta$ luciferase reporter plasmid $(0.1 \mu \mathrm{g})$ and USP17 expression plasmid $(0.3$ $\mu \mathrm{g})$. At $18 \mathrm{~h}$ after transfection, cells were left uninfected or infected with SeV for $12 \mathrm{~h}$ before luciferase assays were performed. Graphs show mean \pm SD, $n=3$. (B) USP17 inhibits SeV-induced activation of ISRE. The experiments were done similarly as in (A), except that the ISRE luciferase reporter plasmid was used. Graphs show mean \pm SD, $n=3$. (C) USP17 inhibits SeV- and TNF- $\alpha$-induced activation of NF-kB. 293 cells $\left(1 \times 10^{5}\right)$ were transfected with an NF-kB luciferase reporter plasmid $(0.1 \mu \mathrm{g})$ and USP17 expression plasmid $(0.3 \mu \mathrm{g})$. At $18 \mathrm{~h}$ after transfection, cells were treated with TNF- $\alpha(20 \mathrm{ng} / \mathrm{ml})$, or infected with $\mathrm{SeV}$, or left untreated for $12 \mathrm{~h}$ before luciferase assays were performed. Graphs show mean $\pm \mathrm{SD}, n=3$. (D) USP17 deubiquitinates components of virus-triggered signaling pathway. 293 cells $\left(1 \times 10^{6}\right)$ were transfected with the indicated expression plasmids ( $5 \mu \mathrm{g}$ each). $24 \mathrm{~h}$ later, cell lysates were immunoprecipitated (IP) and re-immunoprecipitated with the indicated antibodies; the immunoprecipitates were analyzed by immunoblots with anti-Flag (left upper panel) and anti-HA (right upper panel) antibodies. Expression of transfected plasmids was detected by anti-HA (left lower panel) and anti-Flag (right lower panel). The experiments were repeated three times with similar results. (E) USP17 deubiquitinates cellular proteins. 293 cells $\left(2 \times 10^{5}\right)$ were transfected with HA-tagged ubiquitin $(0.1 \mu \mathrm{g})$ and the indicated expression plasmids $(0.5 \mu \mathrm{g})$; $18 \mathrm{~h}$ later, immunoblot analysis was performed with anti-HA (upper panel) and anti-Flag (lower panel). The experiments were repeated three times with similar results.

tion of endogenous IFNB1 and RANTES genes (Figure $2 \mathrm{C}$ ) and the production of IFN- $\beta$ proteins (Figure $2 \mathrm{D}$ ) in 293 cells. Because USP17 knockdown negatively regulated $\mathrm{SeV}$-triggered type I IFN signaling, we examined the physiological roles of USP17 in cellular antiviral response. In plaque assays, the production of VSV increased in USP17 knockdown cells (Figure 2E).
These data suggest that knockdown of USP17 negatively regulates cellular antiviral response. We also determined whether endogenous USP17 is involved in regulation of virus-induced signaling in other cell types. We found that USP17 knockdown also inhibited SeV-induced activation of the IFN- $\beta$ promoter in A549 and human primary monocyte-derived dendritic cells (DCs) (Figure 2F). In 
A
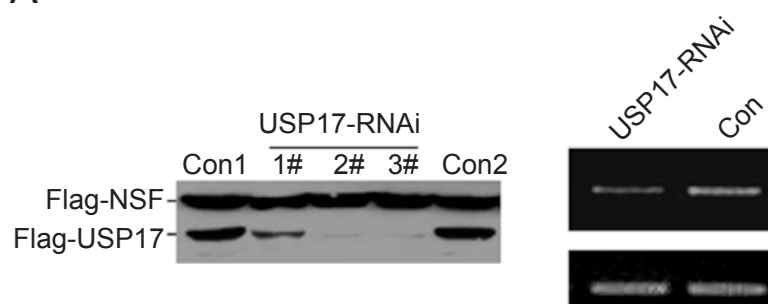

USP17

$-G A P D H$
B

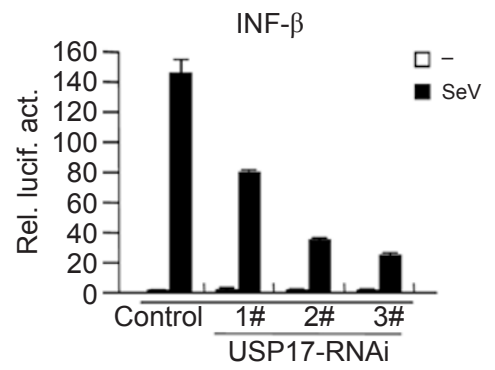

C
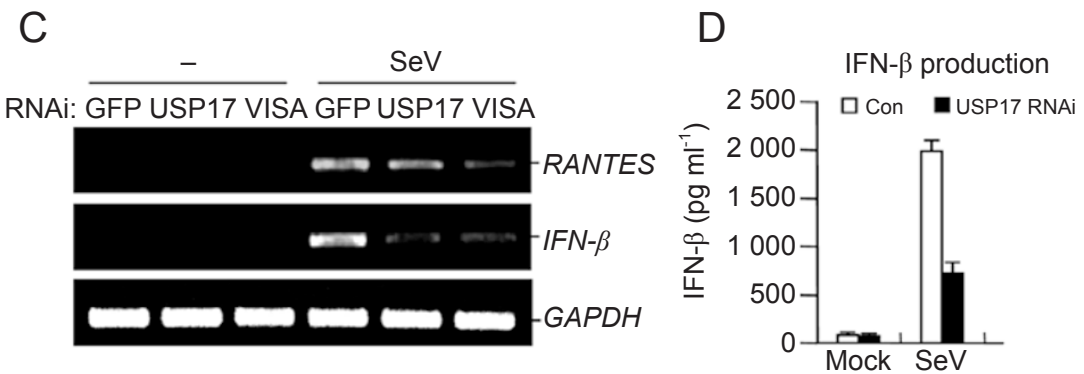

F

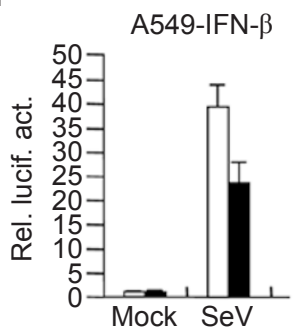

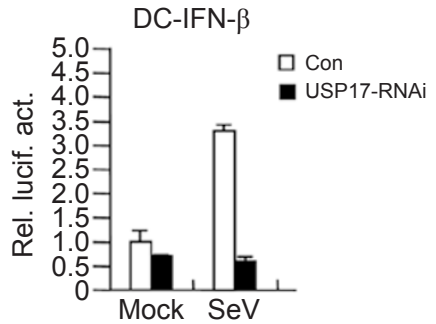

G

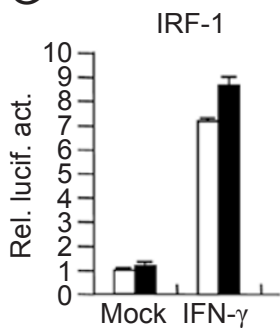

E

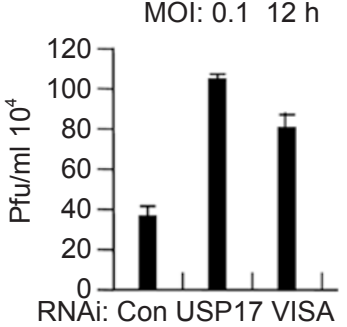

Figure 2 USP17 is required for virus-induced type I IFN signaling. (A) Effects of USP17 RNAi plasmids on expression of transfected and endogenous USP17. In the left panel, 293 cells $\left(2 \times 10^{5}\right)$ were transfected with the indicated expression plasmids $(0.1 \mu \mathrm{g}$ each) and RNAi plasmids $(1 \mu \mathrm{g})$. At $36 \mathrm{~h}$ after transfection, cell lysates were analyzed by immunobloting with anti-Flag. In the right panels, 293 cells $\left(2 \times 10^{5}\right)$ were transfected with control or USP17 RNAi plasmids $(1 \mu \mathrm{g})$; $36 \mathrm{~h}$ after transfection, RT-PCR experiments were performed with specific USP17 (upper) or GAPDH (lower) primers. The experiments were repeated three times with similar results. (B) Effects of USP17 knockdown on SeV-induced activation of the IFN- $\beta$ promoter in 293 cells. 293 cells $\left(1 \times 10^{5}\right)$ were transfected with the IFN- $\beta$ reporter and the indicated RNAi plasmids $(0.5 \mu \mathrm{g}$ each) for 24 $\mathrm{h}$, then infected with SeV or left uninfected for $12 \mathrm{~h}$ before reporter assays were performed. Graphs show mean $\pm \mathrm{SD}, n=3$. (C) Effects of USP17 knockdown on SeV-induced expression of downstream genes. 293 cells $\left(2 \times 10^{5}\right)$ were transfected with the indicated RNAi plasmid (1 $\mu \mathrm{g}$ each). $24 \mathrm{~h}$ after transfection, cells were left uninfected or infected with SeV for $12 \mathrm{~h}$ before RT-PCR was performed. The experiments were repeated three times with similar results. (D) Effects of USP17 knockdown on SeV-induced production of IFN- $\beta$ proteins. 293 cells $\left(2 \times 10^{5}\right)$ were transfected with the indicated RNAi plasmid (1 $\mu \mathrm{g}$ each). Twenty-four hours after transfection, cells were left uninfected or infected with SeV; $1 \mathrm{~h}$ later the medium was changed, and $24 \mathrm{~h}$ later the supernatants were collected for ELLISA. The experiments were repeated three times with similar results. (E) Knockdown of USP17 potentiated VSV replication. 293 cells $\left(1 \times 10^{5}\right)$ were transfected with USP17 RNAi or control plasmid; $24 \mathrm{~h}$ after transfection, the cells were infected with VSV $(\mathrm{MOI}=0.1)$. The supernatants were harvested $24 \mathrm{~h}$ after infection for standard plaque assays. The experiments were repeated three times with similar results. (F) Effects of USP17 knockdown on SeV-induced activation of the IFN- $\beta$ promoter in A549 cells and human primary DCs. A549 cells $\left(1 \times 10^{5}\right)$ or DCs $\left(1 \times 10^{6}\right)$ were transfected with the indicated plasmids. Twenty-four hours after transfection, cells were left uninfected or infected with $\mathrm{SeV}$ for $16 \mathrm{~h}$ before reporter assays were performed. Graphs show mean $\pm \mathrm{SD}, n=3$. (G) Effects of USP17 knockdown on IFN- $\gamma$-induced activation of the IRF-1 promoter and TNF- $\alpha$-induced activation of NF- $\mathrm{B} B$. In the left panel, the experiment was performed similarly as in (B), except that the IRF1 luciferase reporter plasmid was used and transfected cells were treated with IFN- $\gamma(100 \mathrm{ng} / \mathrm{ml})$. In the right panel, the experiment was performed similarly as in (B), except that the NF- $\mathrm{B}$ luciferase reporter plasmid was used, and transfected cells were treated with TNF- $\alpha$ (20 ng/ml). Graphs show mean $\pm \mathrm{SD}, n=3$. 
similar experiments, knockdown of USP17 had no obvious effects on IFN- $\gamma$-induced activation of the IRF1 promoter or on TNF-induced activation of NF- $\mathrm{kB}$ (Figure $2 \mathrm{G}$ ). These data suggest that USP17 is specifically required for virus-triggered signaling in various types of cells.

It has been demonstrated that induction of type I IFNs requires coordinative and cooperative action of the transcription factors IRF3 and NF- $\kappa \mathrm{B}$. We examined whether USP17 is involved in virus-triggered activation of IRF3 and NF- $\kappa B$. Results from reporter assays showed that knockdown of USP17 inhibited SeV-induced activation of ISRE and NF- $\mathrm{BB}$, and the degrees of inhibition were correlated to the knockdown efficiencies of USP17 expression (Figure 3A and 3B). Consistent with these data, knockdown of USP17 inhibited SeV-induced phosphory- lation (Figure 3C) and dimerization (Figure 3D) of IRF3. These data collectively suggest that USP 17 is required for virus-induced activation of IRF3 and NF- $\mathrm{kB}$ and expression of IFN- $\beta$.

\section{USP17 regulates virus-triggered signaling at the RLR} level

Previous studies suggest that cytoplasmic poly(I:C) is recognized by MDA5, while transfected poly(dA:dT) is transcribed into 5'pppssRNA, which is recognized by RIG-I [40]. We next examined whether USP17 is required for RIG-I- or MDA5-mediated signaling. In reporter assays, knockdown of USP17 inhibited both cytoplasmic poly(I:C)- and poly(dA:dT)-induced activation of the IFN- $\beta$ promoter (Figure $4 \mathrm{~A}$ and $4 \mathrm{~B}$ ), whereas knockdown of USP17 had no obvious effects
A

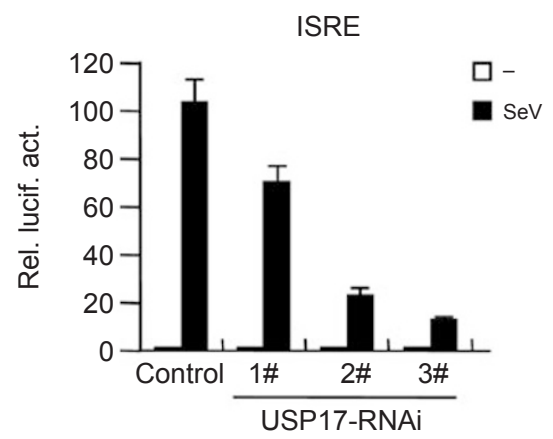

C

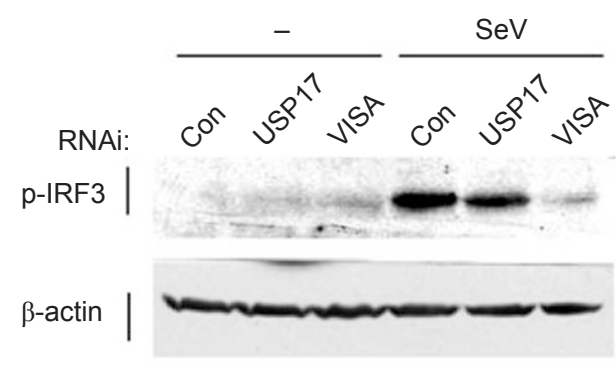

B

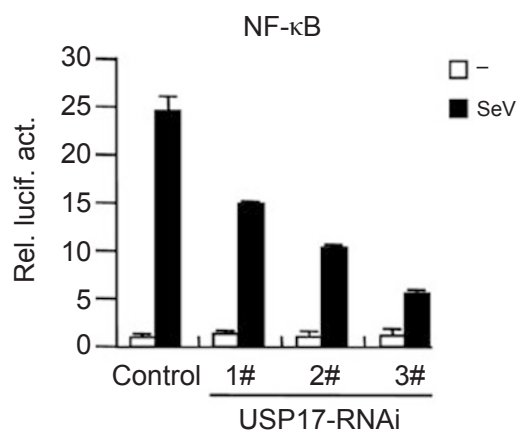

D

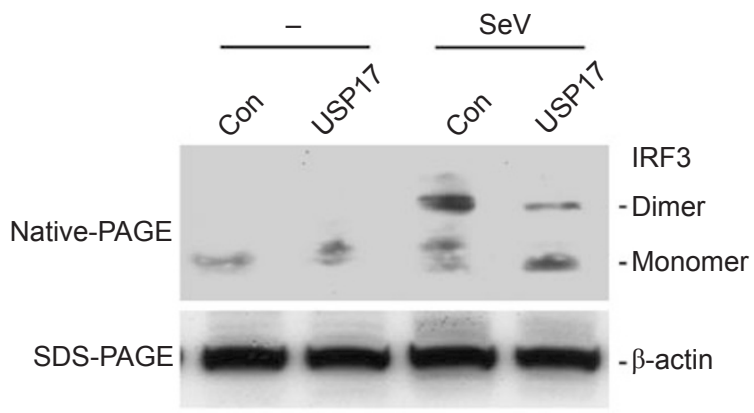

Figure 3 Knockdown of USP17 inhibits SeV-induced activation of ISRE and NF-kB. (A) Effects of USP17 knockdown on $\mathrm{SeV}$-induced activation of the ISRE in 293 cells. The experiment was performed similarly as described in Figure 2B, except that the ISRE luciferase reporter plasmid was used. Graphs show mean \pm SD, $n=3$. (B) Effects of USP17 RNAi plasmids on

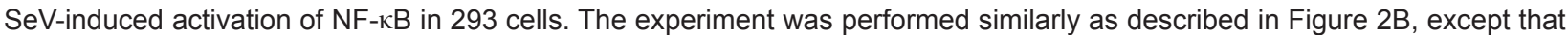
the NF-kB luciferase reporter plasmid was used. Graphs show mean $\pm \mathrm{SD}, n=3$. (C) Effects of USP17 knockdown on SeVinduced phosphorylation of IRF3. 293 cells $\left(2 \times 10^{5}\right)$ were transfected with the indicated RNAi plasmids for $24 \mathrm{~h}$. Cells were then left uninfected or infected with SeV for $6 \mathrm{~h}$ before immunoblots with the indicated antibodies were performed. The experiments were repeated three times with similar results. (D) Effects of USP17 knockdown on SeV-induced dimerization of IRF3. 293 cells $\left(2 \times 10^{5}\right)$ were transfected with the indicated RNAi plasmids for $24 \mathrm{~h}$. Cells were then left uninfected or infected with $\mathrm{SeV}$ for $8 \mathrm{~h}$, then the cells were harvested and lysed in native lysis buffer. The cell lysates were fractionated in $7.5 \%$ native gel and immunoblot analysis was performed with anti-IRF3 (upper panel). For loading controls, the lysates were analyzed by immunoblot with anti- $\beta$-actin. 
A

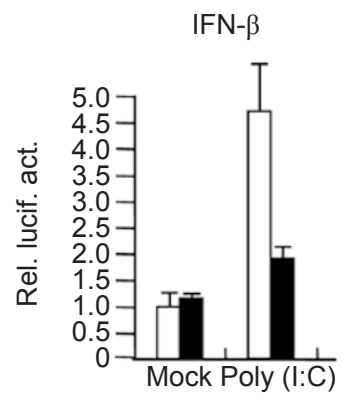

D
B

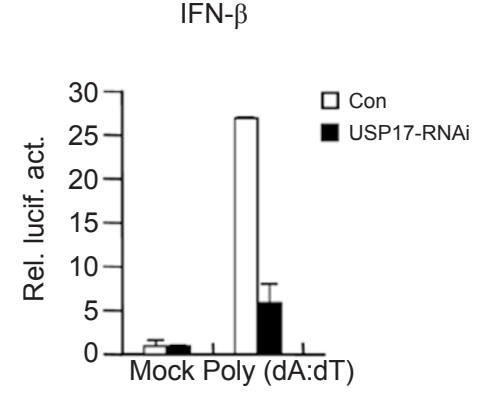

C

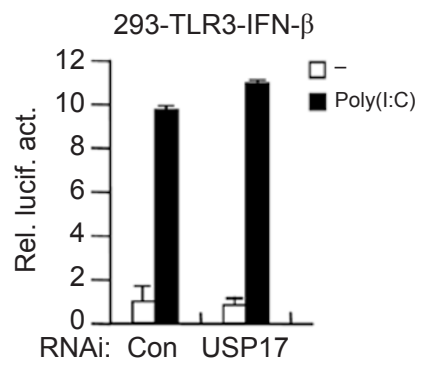

$E$

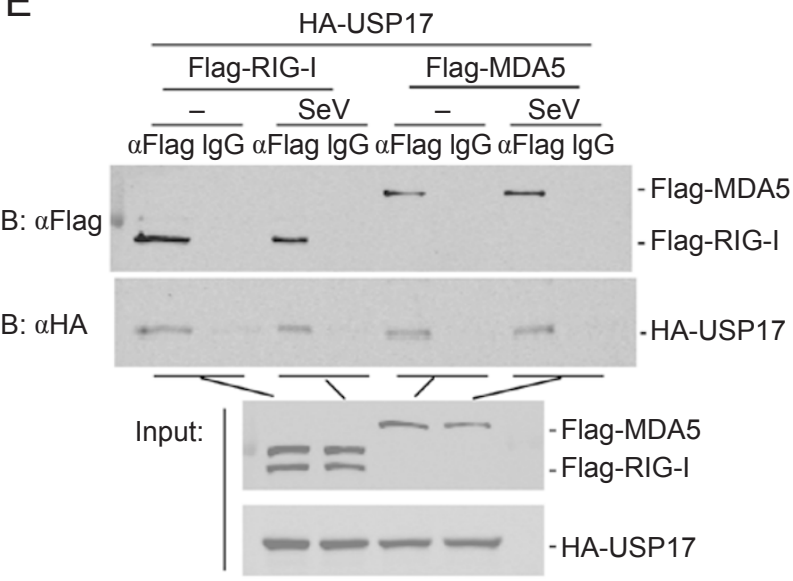

Figure 4 USP17 regulates virus-triggered signaling at the RLR level. (A) Effect of USP17 knockdown on cytoplasmic poly(I:C)-induced activation of the IFN- $\beta$ promoter in 293 cells. 293 cells $\left(1 \times 10^{5}\right)$ were transfected with the indicated RNAi plasmids $(0.5 \mu \mathrm{g}$ each). Twenty-four hours later, cells were further transfected with poly(l:C) $(0.5 \mu \mathrm{g})$ or left untransfected and luciferase assays were performed $24 \mathrm{~h}$ later. Graphs show mean $\pm \mathrm{SD}, n=3$. (B) Effect of USP17 knockdown on cytoplasmic poly(dA:dT)-induced activation of the IFN- $\beta$ promoter in 293 cells. The experiment was done similarly as in (A), except that cells were further transfected with poly(dA:dT) $(0.5 \mu \mathrm{g})$ instead of poly(l:C). (C) Effect of USP17 knockdown on poly(l:C)induced activation of the IFN- $\beta$ promoter in 293-TLR3 cells. 293-TLR3 cells $\left(1 \times 10^{5}\right)$ were transfected with the indicated RNAi plasmids $(0.5 \mu \mathrm{g}$ each). Twenty-four hours later, cells were treated with poly(l:C) $(50 \mu \mathrm{g} / \mathrm{ml})$ or left untreated for $8 \mathrm{~h}$ before luciferase assays were performed. Graphs show mean \pm SD, $n=3$. (D) Knockdown of USP17 inhibited RIG-I- and MDA5-induced activation of the IFN- $\beta$ promoter. 293 cells $\left(1 \times 10^{5}\right)$ were transfected with USP17 RNAi or control plasmid $(0.5$ $\mu \mathrm{g}$ each); $24 \mathrm{~h}$ later, cells were further transfected with the indicated expression plasmids and the IFN- $\beta$ luciferase plasmid before luciferase assays were performed. Graphs show mean \pm SD, $n=3$. (E) USP17 interacted with RIG-I and MDA5. 293 cells $\left(2 \times 10^{6}\right)$ were transfected with the indicated expression plasmids; $20 \mathrm{~h}$ later, cells were infected or uninfected with SeV for $4 \mathrm{~h}$ before coimmunoprecipitations were performed with the indicated antibodies.

on poly(I:C)-induced TLR3-mediated activation of the IFN- $\beta$ promoter (Figure $4 \mathrm{C}$ ), suggesting that USP17 is required for both RIG-I- and MDA5-mediated signaling.

We further determined the molecular order of the involvement of USP17 in virus-triggered signaling. In reporter assays, knockdown of USP17 inhibited RIG-Iand MDA5-induced but not downstream components VISA-, MITA-, TBK1- and IKKE-induced activation of the IFN- $\beta$ promoter (Figure 4D). Interestingly, results from our transfection and coimmunoprecipitation assays suggested that USP17 was associated with RIG-I and MDA5 but not with VISA, MITA or TBK1 in the pres- ence or absence of viral infection (Figure 4E and data not shown). These results suggest that USP17 regulates virus-triggered signaling at the RLR level.

Knockdown of USP17 potentiates the ubiquitination levels of RIG-I and MDA5

It is reported that regulation of ubiquitination represents a critical mechanism for the control of virustriggered production of type I IFNs. USP17 has deubiquitinating enzymatic activity and functions at the RLR level; therefore, it is possible that USP17 regulates ubiquitination of RLRs. To examine this possibility, we 
performed transfection and ubiquitination assays. Coimmunoprecipitation and immunoblot analysis showed that knockdown of USP17 potentiated the ubiquitination levels of RIG-I and MDA5 (Figure 5A). In parallel experiments, knockdown of USP17 did not have obvious effects on ubiquitination of IKK $\varepsilon$ and MITA (Figure 5A), suggesting the specific effects of endogenous USP17 on deubiquitination of RIG-I and MDA5. Furthermore, knockdown of USP17 potentiated both K63- and K48linked ubiquitination of RIG-I (Supplementary information, Figure S2). Previously, it has been shown that viral infection or IFN- $\alpha$ treatment causes ubiquitination of RIG-I [30]. In our experiments, knockdown of USP17 potentiated ubiquitination of endogenous RIG-I after viral infection (Figure 5B). In addition, knockdown of USP17 also inhibited SeV-induced expression of RIG-I and MDA5 (Supplementary information, Figure S3). Taken together, these data suggest that USP17 regulates virus-induced type I IFN signaling through deubiquitination of RIG-I and MDA5.

\section{Discussion}

Ubiquitination and deubiquitination play important roles in regulating virus-triggered induction of type I IFNs. In this study, we screened a panel of deubiquitination enzymes and identified USP17 as a modulator of virus-triggered type I IFN induction pathways.

In an overexpression system, USP17 strongly removed polyubiquitin chains conjugated to all examined signaling components of the virus-triggered signaling pathways, such as MDA-5, RIG-I, VISA, MITA, IKK $\varepsilon$ and TRAF3. Consistent with previous reports that the ubiquitination of some of these proteins, such as RIG-I and TRAF3, is required for virus-triggered induction of type I IFNs, overexpression of USP17 potently inhibited $\mathrm{SeV}$-triggered activation of ISRE and NF- $\kappa \mathrm{B}$ as well as the IFN- $\beta$ promoter. However, the inhibitory effects of USP17 overexpression on virus-triggered signaling are probably artificial because overexpression of USP17 caused global deubiquitination of cellular proteins and/or cytosolic synthesized polyubiquitin chains.

To determine whether USP17 plays a specific and physiological role in virus-triggered signaling, we investigated the effects of USP17 knockdown on virus-triggered signaling. Knockdown of endogenous USP17 attenuated virus-, cytoplasmic poly(I:C)- and poly(dA:dT)induced activation of the IFN- $\beta$ promoter. Further analysis indicated that knockdown of USP17 inhibited SeV-induced phosphorylation and dimerization of IRF3 and expression of IFNBI and RANTES genes, as well as cellular antiviral responses. These results suggest that
A

$\begin{array}{rcccccccc}\text { Flag-RIG-I } & + & + & - & - & - & - & - & - \\ \text { Flag-MDA5 } & - & - & + & + & - & - & - & - \\ \text { Flag-IKKE } & - & - & - & - & + & + & - & - \\ \text { Flag-MITA } & - & - & - & - & - & - & + & + \\ \text { USP17-RNAi } & - & + & - & + & - & + & - & + \\ \text { HA-Ubi } & + & + & + & + & + & + & + & +\end{array}$

B

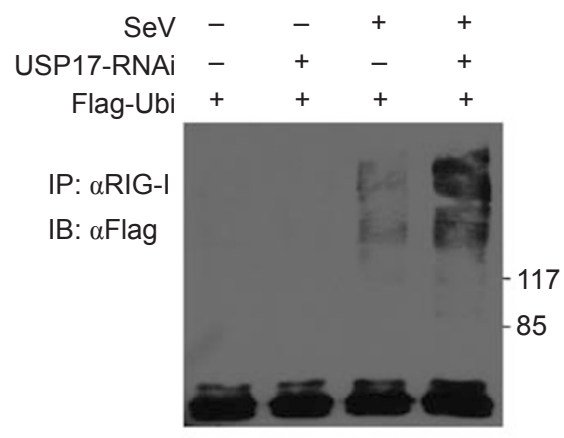

IP: $\alpha$ RIG-I
IB: $\alpha R I G-1$

Figure 5 Knockdown of USP17 potentiates the ubiquitination levels of RIG-I and MDA5. (A) Knockdown of USP17 potentiates ubiquitination of RIG-I and MDA5 in the overexpression system. 293 cells $\left(\sim 1 \times 10^{6}\right)$ were transfected with the indicated expression (5 $\mu \mathrm{g}$ each) and RNAi plasmids (10 $\mu \mathrm{g}$ each). Twenty-four hours after transfection, cell lysates were immunoprecipitated and re-immunoprecipitated with antiFlag. The immunoprecipitates were analyzed by immunoblots with anti-HA (upper panel) or anti-Flag (lower panel). The experiments were repeated three times with similar results. (B) Knockdown of USP17 potentiates SeV-induced ubiquitination of RIG-I. 293 cells $\left(\sim 1 \times 10^{6}\right)$ were transfected with Flag-tagged ubiquitin combined with USP17 RNAi or control plasmids; $24 \mathrm{~h}$ later, cells were left uninfected or infected with $\mathrm{SeV}$ for $24 \mathrm{~h}$, then cell lysates were immunoprecipitated and re-immunoprecipitated with anti-RIG-I. The immunoprecipitates were analyzed by western blots with anti-Flag (upper panel) and anti-RIG-I (lower panel). The experiments were repeated three times with similar results. 
endogenous USP17 is required for virus-triggered induction of type I IFNs.

To investigate at which level USP17 functions, we performed three lines of experiments. In reporter assays, knockdown of endogenous USP17 inhibited RIG-I- and MDA5-induced activation of the IFN- $\beta$ promoter, but not the downstream components VISA-, TBK1- and IKKEinduced activation of the IFN- $\beta$ promoter. Second, our coimmunoprecipitation and immunoblot assays demonstrated that USP17 interacted with RIG-I and MDA5 but not with VISA, MITA or TBK1. In addition, knockdown of endogenous USP17 enhanced the ubiquitination of RIG-I and MDA5, but not that of MITA and IKKe. These results suggest that USP17 regulates virus-induced type I IFN signaling through deubiquitination of RIG-I and MDA5.

Ubiquitination and deubiquitination of RLRs play important roles in regulating virus-induced type I IFN signaling. For example, two ubiquitin E3 enzymes, TRIM25 and REUL (also called RNF135 and Riplet), have been demonstrated to catalyze K63-linked polyubiquitinations of RIG-I, which are critical for activation of RIG-I and signaling to downstream components [30-32]. Two DUBs, A20 and CYLD, have been demonstrated to remove the K63-linked polyubiquitin chains from RIG-I and thereby negatively regulate virus-induced type I IFN signaling $[36,37]$. On the other hand, RNF125 catalyzes K48-linked polyubiquitination of RIG-I and MDA5 on their N-terminal caspase recruitment domains (CARD) and these ubiquitinations cause proteasome-dependent degradation of RIG-I and MDA5, thereby negatively regulating virus-induced type I IFN signaling [28]. In light of these observations, it is possible that USP17 causes removal of K48-linked polyubiquitin chains from RIG-I and MDA5, a step important for preventing the degradation of RIG-I and MDA5 and therefore promoting virus-trigged type I IFN signaling. In this context, we observed that knockdown of USP17 inhibited virusinduced expression of RIG-I and MDA5. However, the exact targeted lysine residues remain to be identified. Taken together, the identification of USP17 as a DUB for RIG-I and MDA5 helps to understand how the early steps of virus-triggered signaling are regulated.

\section{Materials and Methods}

\section{Reagents and antibodies}

TNF- $\alpha$ (R\&D Systems), IFN- $\gamma$ (R\&D Systems), poly(I:C) (Invitrogen), poly(dA:dT) (Amersham Biosciences), mouse monoclonal antibodies against Flag (M2), HA epitopes and $\beta$-actin (Sigma), and rabbit polyclonal antibody against phospho-IRF3 (Cell Signaling Technology) were purchased from the indicated manufacturers. $\mathrm{SeV}$ and VSV were previously described [41].

\section{Constructs}

NF- $\kappa$ B, ISRE, IFN- $\beta$, TK and IRF1 luciferase reporter plasmids and mammalian expression plasmids for Flag- or HA-tagged RIG-I, MDA5, VISA, MITA, TBK1, IKK $\varepsilon$ and IRF3 were previously described $[11,15,42]$. Mammalian expression plasmids for Flag-tagged USP17, USP5, DUBA and HA-tagged ubiquitin mutations were constructed by standard molecular biology techniques.

\section{Expression cloning}

The expression clones encoding DUBs were transfected into 293 cells together with the IFN- $\beta$ luciferase reporter by standard calcium phosphate precipitation; to normalize for transfection efficiency, TK luciferase reporter plasmid was added to each transfection. Sixteen hours after transfection, cells were infected with $\mathrm{SeV}$ or left uninfected for $8 \mathrm{~h}$. The clones that inhibited SeV-triggered activation of the IFN- $\beta$ promoter were isolated and validated by repeating the reporter assays.

\section{Coimmunoprecipitation and immunoblot analysis}

Transfected 293 cells from each 10-cm dish were lysed in $1 \mathrm{ml}$ NP-40 lysis buffer (20 mM Tris-HCl, $\mathrm{pH}$ 7.4-7.5, $150 \mathrm{mM} \mathrm{NaCl}$, $1 \mathrm{mM}$ EDTA, $1 \% \mathrm{NP}-40,10 \mu \mathrm{g} / \mathrm{ml}$ aprotinin, $10 \mu \mathrm{g} / \mathrm{ml}$ leupeptin, $1 \mathrm{mM}$ phenylmethylsulfonyl fluoride). For each immunoprecipitation, $0.8 \mathrm{ml}$ cell lysate was incubated with $0.5 \mu \mathrm{g}$ of the indicated antibody and $25 \mu \mathrm{l}$ of $50 \%$ slurry of GammaBind G Plus-Sepharose (Amersham Biosciences) at $4{ }^{\circ} \mathrm{C}$ for $2 \mathrm{~h}$. Then, the sepharose beads were washed three times with $1 \mathrm{ml}$ lysis buffer containing $500 \mathrm{mM} \mathrm{NaCl}$. The precipitates were subjected to $8 \%$ SDS-PAGE and subsequent immunoblot analysis was performed with the indicated antibodies.

\section{Re-immunoprecipitation experiments}

For detection of ubiquitinated proteins, re-immunoprecipitation experiments were performed. First-round immunoprecipitation was performed as above. The immunoprecipitates were re-extracted in lysis buffer containing 1\% SDS and denatured by heating for $5 \mathrm{~min}$. The supernatants were diluted with regular lysis buffer till the concentration of SDS was decreased to $0.1 \%$. The diluted supernatants were re-immunoprecipitated with the indicated antibodies and the immunoprecipitates were analyzed by immunoblots with an antibody against ubiquitin or tag fused to ubiquitin.

\section{Generation and transfection of human primary DCs}

Peripheral blood monocytes (PBMCs) were isolated from healthy human peripheral blood by density gradient separation using Ficoll-Paque (GE Healthcare) following the manufacturer's instructions. The isolated PBMCs were incubated with human CD14-specific antibody conjugated to paramagnetic microbeads (Miltenyi Biotec) and the $\mathrm{CD} 14^{+}$monocytes were isolated on the LS columns (Miltenyi Biotec). The monocytes were suspended in RPMI 1640 supplemented with 10\% heat-inactivated FBS (ExCell), $1 \%$ nonessential amino acids and $1 \mathrm{mM}$ sodium pyruvate. For generation of DCs, the $\mathrm{CD} 14^{+}$monocytes were treated with recombinant human (rh) GM-CSF (50 ng/ml) plus rhIL-4 (100 $\mathrm{ng} / \mathrm{ml}$ ) (PeproTech Inc.) for 6 days. The media with the cytokines were changed every other day during the differentiation period. Differentiated DCs were harvested and transfected using the human DC nucleofactor kit (Amaxa). Immediately after transfection, cells were resuspended in the cytokine-containing media. Twenty- 
four hours after transfection, cells were infected with $\mathrm{SeV}$ for $16 \mathrm{~h}$ before reporter assays were performed.

\section{RNAi experiments}

Double-strand oligonucleotides corresponding to the target sequences were cloned into the pSuper.Retro RNAi plasmid (Oligoengine Inc.). The following sequences were targeted for human USP17 cDNA:\#1: 5'-GAT CCA AAG GGA AGA ACA A-3'; \#2: 5'-GAT CAT CAC TCT AAG GAC A-3'; \#3: 5'-GAG ATT CTC CGA TGT CAC A-3'.

\section{RT-PCR and ELISA}

Total RNA from transfected 293 cells was isolated using Trizol reagent (Invitrogen); after reverse transcription with oligo(dT) primer using a RevertAidTM First Strand cDNA Synthesis Kit (Fermentas), aliquots of products were subjected to PCR using gene-specific primers as follows: $I F N-\beta, 5^{\prime}$-CAG CAA TTT TCA GTG TCA GAA GCT-3' and 5'-CAG TGA CTG TAC TCC TTG GCC TT-3'; Rantes, 5'-ATG AAG GTC TCC GCG GCA CGC CT3' and 5'-CTA GCT CAT CTC CAA AGA GTT G-3'; GAPDH, 5'CGG AGT CAA CGG ATT TGG TCG-3' and 5'-AGC CTT CTC CAT GGT GGT GAA G-3'; and USP17, 5'-CAC AGT GAG AGT GTG TCA AGA-3' and 5'-TCA CTG GCA CAC AAG CAG AGC CC-3'. The supernatants from infected cells were collected and subjected to ELISA using a commercial human IFN- $\beta$ kit (PBL Biomedical laboratories).

\section{VSV plaque assay}

The 293 cells $\left(1 \times 10^{5}\right)$ were transfected with the indicated RNAi plasmids for $24 \mathrm{~h}$ prior to VSV infection $(\mathrm{MOI}=0.1) ; 1 \mathrm{~h}$ later, cells were washed three times with PBS and then medium was added. The supernatants were collected $12 \mathrm{~h}$ later and diluted $1: 10^{4}$ to infect confluent BHK21 cells cultured in 24-well plates; 1 $\mathrm{h}$ later, the supernatant was removed and $2 \%$ methylcellulose was overplayed. At 2 days post infection, the overplay was removed and cells were fixed with $4 \%$ formaldehyde for $1 \mathrm{~h}$ and stained with $0.2 \%$ Crystal violet in $20 \%$ methanol. Plaques were counted, averaged and multiplied by the dilution factor to determine the viral titer as PFU/ml.

\section{Acknowledgments}

We thank the members of our laboratory for discussions. This work was supported by grants from the National Basic Research Program of China (973 Program) (2006CB504301 and 2010CB911802) and the National Natural Science Foundation of China (30921001 and 30700417).

\section{References}

1 Akira S, Uematsu S, Takeuchi O. Pathogen recognition and innate immunity. Cell 2006; 124:783-801.

2 Hiscott J. Convergence of the NF-kappaB and IRF pathways in the regulation of the innate antiviral response. Cytokine Growth Factor Rev 2007; 18:483-490.

3 Honda K, Takaoka A, Taniguchi T. Type I interferon [corrected] gene induction by the interferon regulatory factor family of transcription factors. Immunity 2006; 25:349-360.

4 Alexopoulou L, Holt AC, Medzhitov R, Flavell RA. Recog- nition of double-stranded RNA and activation of NF- $\kappa$ B by Toll-like receptor 3. Nature 2001; 413:732-738.

5 Kato H, Takeuchi O, Sato S, et al. Differential roles of MDA5 and RIG-I helicases in the recognition of RNA viruses. Nature 2006; 441:101-105.

6 Pichlmair A, Schulz O, Tan CP, et al. RIG-I-mediated antiviral responses to single-stranded RNA bearing 5'-phosphates. Science 2006; 314:997-1001.

7 Han KJ, Su X, Xu LG, et al. Mechanisms of the TRIFinduced interferon-stimulated response element and NF$\kappa \mathrm{B}$ activation and apoptosis pathways. J Biol Chem 2004; 279:15652-15661.

8 Oshiumi H, Sasai M, Shida K, et al. TIR-containing adapter molecule (TICAM)-2, a bridging adapter recruiting to tolllike receptor 4 TICAM-1 that induces interferon-beta. J Biol Chem 2003; 278:49751-49762.

9 Yamamoto M, Sato S, Hemmi H, et al. Role of adaptor TRIF in the MyD88-independent toll-like receptor signaling pathway. Science 2003; 301:640-643.

10 Yamamoto $\mathrm{M}$, Sato S, Mori K, et al. Cutting edge: a novel Toll/IL-1 receptor domain-containing adapter that preferentially activates the IFN- $\beta$ promoter in the Toll-like receptor signaling. J Immunol 2002; 169:6668-6672.

$11 \mathrm{Xu} \mathrm{LG}$, Wang YY, Han KJ, et al. VISA is an adapter protein required for virus-triggered IFN- $\beta$ signaling. Mol Cell 2005; 19:727-740.

12 Seth RB, Sun L, Ea CK, Chen ZJ. Identification and characterization of MAVS, a mitochondrial antiviral signaling protein that activates NF- $\mathrm{KB}$ and IRF 3. Cell 2005; 122:669-682.

13 Kawai T, Takahashi K, Sato S, et al. IPS-1, an adaptor triggering RIG-I- and Mda5-mediated type I interferon induction. Nat Immunol 2005; 6:981-988.

14 Meylan E, Curran J, Hofmann K, et al. Cardif is an adaptor protein in the RIG-I antiviral pathway and is targeted by hepatitis C virus. Nature 2005; 437:1167-1172.

15 Zhong B, Yang Y, Li S, et al. The adaptor protein MITA links virus-sensing receptors to IRF3 transcription factor activation. Immunity 2008; 29:538-550.

16 Ishikawa H, Barber GN. STING is an endoplasmic reticulum adaptor that facilitates innate immune signalling. Nature 2008; 455:674-678.

17 Chiu YH, Macmillan JB, Chen ZJ. RNA polymerase III detects cytosolic DNA and induces type I interferons through the RIG-I pathway. Cell 2009; 138:576-591.

18 Ablasser A, Bauernfeind F, Hartmann G, et al. RIG-I-dependent sensing of poly(dA:dT) through the induction of an RNA polymerase III-transcribed RNA intermediate. Nat Immunol 2009; 10:1065-1072.

19 Nijman SM, Luna-Vargas MP, Velds A, et al. A genomic and functional inventory of deubiquitinating enzymes. Cell 2005; 123:773-786.

20 Song L, Rape M. Reverse the curse--the role of deubiquitination in cell cycle control. Curr Opin Cell Biol 2008; 20:156163.

21 Schmidt M, Hanna J, Elsasser S, Finley D. Proteasomeassociated proteins: regulation of a proteolytic machine. Biol Chem 2005; 386:725-737.

22 Komada M. Controlling receptor downregulation by ubiquitination and deubiquitination. Curr Drug Discov Technol 
2008; 5:78-84.

23 Guterman A, Glickman MH. Deubiquitinating enzymes are IN/(trinsic to proteasome function). Curr Protein Pept Sci 2004; 5:201-211.

24 Daniel JA, Grant PA. Multi-tasking on chromatin with the SAGA coactivator complexes. Mutat Res 2007; 618:135-148.

25 Kayagaki N, Phung Q, Chan S, et al. DUBA: a deubiquitinase that regulates type I interferon production. Science 2007; 318:1628-1632.

26 Adhikari A, Xu M, Chen ZJ. Ubiquitin-mediated activation of TAK1 and IKK. Oncogene 2007; 26:3214-3226.

27 Pickart CM. Mechanisms underlying ubiquitination. Annu Rev Biochem 2001; 70:503-533.

28 Arimoto K, Takahashi H, Hishiki T, et al. Negative regulation of the RIG-I signaling by the ubiquitin ligase RNF125. Proc Natl Acad Sci USA 2007; 104:7500-7505.

29 Gack MU, Kirchhofer A, Shin YC, et al. Roles of RIG-I $\mathrm{N}$-terminal tandem CARD and splice variant in TRIM25mediated antiviral signal transduction. Proc Natl Acad Sci USA 2008; 105:16743-16748.

30 Gack MU, Shin YC, Joo CH, et al. TRIM25 RING-finger E3 ubiquitin ligase is essential for RIG-I-mediated antiviral activity. Nature 2007; 446:916-920.

31. Gao D, Yang YK, Wang RP, et al. REUL is a novel E3 ubiquitin ligase and stimulator of retinoic-acid-inducible gene-I. PLoS One 2009; 4:e5760.

32 Oshiumi H, Matsumoto M, Hatakeyama S, Seya T. Riplet/ RNF135, a RING finger protein, ubiquitinates RIG-I to promote interferon- $\beta$ induction during the early phase of viral infection. J Biol Chem 2009; 284:807-817.

33 Shi M, Deng W, Bi E, et al. TRIM30 alpha negatively regulates TLR-mediated NF- $\kappa \mathrm{B}$ activation by targeting TAB2 and
TAB3 for degradation. Nat Immunol 2008; 9:369-377.

34 Zhang M, Tian Y, Wang RP, et al. Negative feedback regulation of cellular antiviral signaling by RBCK1-mediated degradation of IRF3. Cell Res 2008; 18:1096-1104.

35 Zhong B, Zhang L, Lei C, et al. The ubiquitin ligase RNF5 regulates antiviral responses by mediating degradation of the adaptor protein MITA. Immunity 2009; 30:397-407.

36 Lin R, Yang L, Nakhaei P, et al. Negative regulation of the retinoic acid-inducible gene I-induced antiviral state by the ubiquitin-editing protein A20. J Biol Chem 2006; 281:20952103.

37 Zhang $\mathrm{M}, \mathrm{Wu} \mathrm{X}$, Lee AJ, et al. Regulation of IкB kinaserelated kinases and antiviral responses by tumor suppressor CYLD. J Biol Chem 2008; 283:18621-18626.

38 Shin JM, Yoo KJ, Kim MS, Kim D, Baek KH. Hyaluronanand RNA-binding deubiquitinating enzymes of USP17 family members associated with cell viability. BMC Genomics 2006; 7:292.

39 Burrows JF, Kelvin AA, McFarlane C, et al. USP17 regulates Ras activation and cell proliferation by blocking RCE1 activity. J Biol Chem 2009; 284:9587-9595.

40 Kato H, Takeuchi O, Mikamo-Satoh E, et al. Length-dependent recognition of double-stranded ribonucleic acids by retinoic acid-inducible gene-I and melanoma differentiationassociated gene 5. J Exp Med 2008; 205:1601-1610.

41 Diao F, Li S, Tian Y, et al. Negative regulation of MDA5but not RIG-I-mediated innate antiviral signaling by the dihydroxyacetone kinase. Proc Natl Acad Sci USA 2007; 104:11706-11711.

42 Huang J, Liu T, Xu LG, et al. SIKE is an IKK epsilon/TBK1associated suppressor of TLR3- and virus-triggered IRF-3 activation pathways. EMBO J 2005; 24:4018-4028.

(Supplementary information is linked to the online version of the paper on the Cell Research website.) 